\title{
An upper bound for the pseudoisotopy stable range
}

\author{
Oscar Randal-Williams ${ }^{1}$ (D)
}

Received: 15 July 2016 / Revised: 16 November 2016 / Published online: 1 December 2016

C) The Author(s) 2016. This article is published with open access at Springerlink.com

\begin{abstract}
We prove that the pseudoisotopy stable range for manifolds of dimension $2 n$ can be no better than $(2 n-2)$. In order to do so, we define new characteristic classes for block bundles, extending our earlier work with Ebert, and prove their nontriviality. We also explain how similar methods show that $\operatorname{Top}(2 n) / \mathrm{O}(2 n)$ is rationally $(4 n-5)$-connected.
\end{abstract}

For a smooth manifold $M$, possibly with boundary, the space of smooth pseudoisotopies (also known as concordances) is $P(M):=\operatorname{Diff}(M \times[0,1] \mathrm{rel} M \times\{0\})$, that is, the space of diffeomorphisms of the cylinder $M \times[0,1]$ which keep one end fixed. There is a canonical map

$$
P(M) \longrightarrow P(M \times I)
$$

given by crossing with the interval $I$ (and unbending corners), and the (smooth) pseudoisotopy stable range is the function

$\phi(n):=\max \{k \in \mathbb{N} \mid(0.1)$ is $k$-connected for all manifolds $M$ of dimension $\geq n\}$.

The main theorem concerning this function is due to Igusa [16], and says that

$$
\phi(n) \geq \min \left\{\frac{n-7}{2}, \frac{n-4}{3}\right\}
$$

In this note we establish the following upper bound for this function.

Theorem A $\phi(2 n) \leq 2 n-2$ as long as $2 n \geq 6$.

\footnotetext{
$凶$ Oscar Randal-Williams

o.randal-williams@dpmms.cam.ac.uk

1 Centre for Mathematical Sciences, Wilberforce Road, Cambridge CB3 0WB, UK
} 
To explain our approach, let $W_{g, 1}:=\#^{g} S^{n} \times S^{n} \backslash \operatorname{int}\left(D^{2 n}\right)$ with $2 n \geq 6$, and consider the fibration sequence

$$
\frac{\widetilde{\operatorname{Diff}_{\partial}}\left(W_{g, 1}\right)}{\operatorname{Diff}_{\partial}\left(W_{g, 1}\right)} \longrightarrow B \operatorname{Diff}_{\partial}\left(W_{g, 1}\right) \stackrel{i}{\longrightarrow} B \widetilde{\operatorname{Diff}_{\partial}}\left(W_{g, 1}\right)
$$

from the classifying space of the group of diffeomorphisms of $W_{g, 1}$ to the classifying space of the group of block diffeomorphisms of $W_{g, 1}$. The rational cohomology of $B \operatorname{Diff}_{\partial}\left(W_{g, 1}\right)$ has been computed for $g \gg 0$ by Galatius and the author in $[12,13]$; the rational cohomology of $\widehat{B \operatorname{Diff}_{\partial}}\left(W_{g, 1}\right)$ has been computed for $g \gg 0$ by Berglund and Madsen in [1,2] and in a forthcoming revision of [2]. Ebert and the author have shown in [8] that the map $i$ is surjective on rational cohomology in the stable range.

Our approach to Theorem A is motivated by forthcoming work of Berglund and Madsen, in which they show that the map induced by $i$ on rational cohomology is injective in degrees $*<2 n$ and $g \gg 0$, and more importantly for our current purpose they show that this is sharp, in the following sense.

Proposition B (Berglund-Madsen) For $g \gg 0$,

$$
\operatorname{Ker}\left(i^{*}: H^{2 n}\left(\widetilde{B \operatorname{Diff}_{\partial}}\left(W_{g, 1}\right) ; \mathbb{Q}\right) \rightarrow H^{2 n}\left(B \operatorname{Diff}_{\partial}\left(W_{g, 1}\right) ; \mathbb{Q}\right)\right) \neq 0
$$

This has implications for the Serre spectral sequence of (0.2), and it is this that we shall exploit to prove Theorem A. As Proposition B is central to our argument, and its proof is not yet available, in Sects. 2 and 3 we will give an independent proof of it, which works for all $g \geq 1$ and does not require the computation of both groups. It consists of defining Mumford-Morita-Miller classes for block bundles, which extend those that we have already defined with Ebert in [8], and then showing that a certain such class - namely $\tilde{\kappa}_{e^{2}}-\tilde{\kappa}_{p_{n}}$, which is easily seen to lie in the kernel (0.3) - is not trivial. The construction of these classes and their non-triviality may be of interest independently of Theorem A.

Finally, in Sect. 4 we show how similar methods can be used to show that the space $\operatorname{Top}(2 n) / \mathrm{O}(2 n)$ is rationally $(4 n-5)$-connected as long as $2 n>4$.

\section{Proof of Theorem A}

By the work of Weiss-Williams [22, Theorem A], there is a certain map

$$
\frac{\widetilde{\operatorname{Diff}_{\partial}}\left(W_{g, 1}\right)}{\operatorname{Diff}_{\partial}\left(W_{g, 1}\right)} \longrightarrow \Omega^{\infty}\left(S_{+}^{\infty} \wedge \mathbb{Z} / 2 \Omega \mathbf{W h}_{s}^{\text {Diff }}\left(W_{g, 1}\right)\right)
$$

which is $(\phi(2 n)+1)$-connected. The $(\mathbb{Z} / 2-)$ spectrum $\mathbf{W h}_{s}^{\text {Diff }}\left(W_{g, 1}\right)$ is the 1-connected cover of the (smooth) Whitehead spectrum $\mathbf{W h}^{\text {Diff }}\left(W_{g, 1}\right)$, which in turn is related to Waldhausen's algebraic $K$-theory of spaces by a (split) cofibre sequence of spectra 


$$
\Sigma_{+}^{\infty} W_{g, 1} \longrightarrow \mathbf{A}\left(W_{g, 1}\right) \longrightarrow \mathbf{W h}^{\text {Diff }}\left(W_{g, 1}\right)
$$

This identification requires the stable parameterised $h$-cobordism theorem [20].

Our strategy is then as follows. We use a theorem of Hsiang-Staffeldt to compute the spectrum cohomology $H^{*}\left(\mathbf{W h}^{\text {Diff }}\left(W_{g, 1}\right) ; \mathbb{Q}\right)$ in degrees $* \leq 2 n$. We take care to compute this as a representation of the mapping class group $\Gamma_{g, 1}$ of $W_{g, 1}$, in terms of the standard representation

$$
H_{g}:=H_{n}\left(W_{g, 1} ; \mathbb{Q}\right)
$$

of $\Gamma_{g, 1}$. The spectrum cohomology of $S_{+}^{\infty} \wedge_{\mathbb{Z} / 2} \Omega \mathbf{W h}_{S}^{\text {Diff }}\left(W_{g, 1}\right)$ is then given by truncating, desuspending, and taking $\mathbb{Z} / 2$-invariants, and the cohomology of $\Omega^{\infty}\left(S_{+}^{\infty} \wedge_{\mathbb{Z} / 2} \Omega \mathbf{W h}_{s}^{\text {Diff }}\left(W_{g, 1}\right)\right)$ is the free graded-commutative algebra on the result.

We now suppose for a contradiction that $\phi(2 n) \geq 2 n-1$, so the map (1.1) is $2 n$ connected and hence we have a computation of the rational cohomology of $\frac{\widetilde{\operatorname{Diff}_{\partial}}\left(W_{g, 1}\right)}{\operatorname{Diff}_{\partial}\left(W_{g, 1}\right)}$ in degrees $* \leq 2 n-1$, as a $\Gamma_{g, 1}$-module. We then study the Serre spectral sequence for $(0.2)$, and derive a contradiction.

\subsection{Rational homology of the Whitehead spectrum}

We shall use Corollary 1.2 of Hsiang-Staffeldt [15], which shows that

$$
H_{*}\left(\mathbf{A}\left(W_{g, 1}\right) ; \mathbb{Q}\right)=\pi_{*}\left(\mathbf{A}\left(W_{g, 1}\right)\right) \otimes \mathbb{Q} \cong\left(K_{*}(\mathbb{Z}) \otimes \mathbb{Q}\right) \oplus\left(\Sigma \bar{K}_{a b}\right)
$$

where $K$ is a minimal model for the $\operatorname{dga} C_{*}\left(\Omega W_{g, 1} ; \mathbb{Q}\right), \bar{K}$ denotes the augmentation ideal, which inherits the structure of a graded Lie algebra with bracket given by $[x, y]:=x \cdot y-(-1)^{|x| \cdot|y|} y \cdot x$, and $\bar{K}_{a b}=\bar{K} /[\bar{K}, \bar{K}]$ is the abelianisation of this graded Lie algebra.

As $W_{g, 1}$ is a suspension, the homology of $\Omega W_{g, 1}$ is the tensor algebra on the vector space $H_{g}[n-1]$. In particular it is a free (non-commutative) algebra, so is quasiisomorphic to $C_{*}\left(\Omega W_{g, 1} ; \mathbb{Q}\right)$, and we may take $K=H_{*}\left(\Omega W_{g, 1} ; \mathbb{Q}\right)$ with trivial differential. It follows that $\bar{K}_{a b}$ is the augmentation ideal of the free graded commutative algebra on $H_{g}[n-1]$, that is

$$
\begin{aligned}
\bar{K}_{a b}=\left(H_{g}[n-1]\right) & \oplus\left(\begin{array}{ll}
\operatorname{Sym}^{2}\left(H_{g}\right)[2 n-2] & \text { if } n \text { is odd } \\
\wedge^{2}\left(H_{g}\right)[2 n-2] & \text { if } n \text { is even }
\end{array}\right) \\
& \oplus(\text { terms of degree } \geq 3 n-3) .
\end{aligned}
$$

Let us write

$$
U:= \begin{cases}\operatorname{Sym}^{2}\left(H_{g}\right) & \text { if } n \text { is odd } \\ \wedge^{2}\left(H_{g}\right) & \text { if } n \text { is even. }\end{cases}
$$


Then we have

$$
H_{*}\left(\mathbf{A}\left(W_{g, 1}\right) ; \mathbb{Q}\right) \cong\left(K_{*}(\mathbb{Z}) \otimes \mathbb{Q}\right) \oplus\left(H_{g}[n]\right) \oplus(U[2 n-1])
$$

in degrees $* \leq 2 n$. Applying the cofibre sequence (1.2), we obtain

$$
H_{*}\left(\mathbf{W h}^{\text {Diff }}\left(W_{g, 1}\right) ; \mathbb{Q}\right) \cong\left(\tilde{K}_{*}(\mathbb{Z}) \otimes \mathbb{Q}\right) \oplus(U[2 n-1])
$$

in degrees $* \leq 2 n$. The rational homology of $\mathbf{W h}_{s}^{\text {Diff }}\left(W_{g, 1}\right)$ is therefore the same, as it is already 1-connected. Thus, dualising, we have

$$
H^{*}\left(S_{+}^{\infty} \wedge_{\mathbb{Z} / 2} \Omega \mathbf{W h}_{s}^{\text {Diff }}\left(W_{g, 1}\right) ; \mathbb{Q}\right) \cong\left(\left(\tilde{K}_{*-1}(\mathbb{Z}) \otimes \mathbb{Q}\right) \oplus(U[2 n-2])\right)^{\mathbb{Z} / 2}
$$

in degrees $* \leq 2 n-1$, for some involution. It follows from Farrell-Hsiang [10] (which considers the case $g=0$ ) that this involution acts as -1 on $\tilde{K}_{*-1}(\mathbb{Z}) \otimes \mathbb{Q}$, so this summand does not contribute to the invariants. Thus

$$
H^{*}\left(S_{+}^{\infty} \wedge_{\mathbb{Z} / 2} \Omega \mathbf{W h}_{s}^{\text {Diff }}\left(W_{g, 1}\right) ; \mathbb{Q}\right) \cong(U[2 n-2])^{\mathbb{Z} / 2}
$$

in degrees $* \leq 2 n-1$, for some involution on $U$. Taking the free graded-commutative algebra on this, it follows that

$$
H^{*}\left(\Omega^{\infty}\left(S_{+}^{\infty} \wedge_{\mathbb{Z} / 2} \Omega \mathbf{W h}_{s}^{\text {Diff }}\left(W_{g, 1}\right)\right) ; \mathbb{Q}\right) \cong \mathbb{Q}[0] \oplus(U[2 n-2])^{\mathbb{Z} / 2}
$$

in degrees $* \leq 2 n-1$.

\subsection{The Serre spectral sequence argument}

The Serre spectral sequence for the fibration (0.2) takes the form

$E_{1}^{p, q}=H^{p}\left(\widetilde{B \operatorname{Diff}_{\partial}}\left(W_{g, 1}\right) ; H^{q}\left(\widetilde{\widetilde{\operatorname{Diff}}_{\partial}\left(W_{g, 1}\right)} ; \mathbb{Q}\right)\right) \Longrightarrow H^{p+q}\left(B \operatorname{Diff}_{\partial}\left(W_{g, 1}\right) ; \mathbb{Q}\right)$

Under the assumption that $\phi(2 n) \geq 2 n-1$ we have identified the coefficients in degrees $q \leq 2 n-1$, to be $\mathbb{Q}$ for $q=0$ and to be $V:=U^{\mathbb{Z} / 2}$ for $q=2 n-2$. In order for $(0.3)$ to be possible, we must therefore have a non-trivial differential

$$
d^{2 n-1}: H^{1}\left(\widetilde{B \operatorname{Diff}_{\partial}}\left(W_{g, 1}\right) ; V\right) \longrightarrow H^{2 n}\left(B \widetilde{\operatorname{Diff}_{\partial}}\left(W_{g, 1}\right) ; \mathbb{Q}\right)
$$

In particular, the source must be non-trivial. Note that $H^{1}\left(\widetilde{B \widetilde{D i f f}_{\partial}}\left(W_{g, 1}\right) ; V\right)$ is a summand of $H^{1}\left(\widehat{B \operatorname{Diff}_{\partial}}\left(W_{g, 1}\right) ; U\right)$, so the following will give a contradiction.

Proposition 1.1 $H^{1}\left(\widetilde{B \operatorname{Diff}_{\partial}}\left(W_{g, 1}\right) ; U\right)=0$ for $g \gg 0$. 
Proof The action of $\Gamma_{g, 1}$ on $H_{n}\left(W_{g, 1} ; \mathbb{Z}\right)$ preserves the intersection form, determining a homomorphism

$$
\Gamma_{g, 1} \longrightarrow \begin{cases}O_{g, g}(\mathbb{Z}) & \text { if } n \text { is even } \\ S p_{2 g}(\mathbb{Z}) & \text { if } n \text { is odd. }\end{cases}
$$

This is onto if $n$ is even or $n=1,3,7$, but for the remaining odd $n$ its image is the finite-index subgroup-often denoted $\Gamma_{g}(1,2) \leq S p_{2 g}(\mathbb{Z})$ in the theory of theta functions - of those symplectic matrices which preserve the standard quadratic form, cf. [2, Example 4.2]. Let us write $G$ for the algebraic group $O_{g, g}$ or $S p_{2 g}$, depending on the parity of $n$, and $A_{g} \leq G(\mathbb{Z})$ for the image of this homomorphism. As $S p_{2 g}$ and $S O_{g, g}$ are connected semisimple algebraic groups defined over $\mathbb{Q}$, it follows from a theorem of Borel-Harish-Chandra [5, Theorem 7.8] that $A_{g}$ is a lattice in $G(\mathbb{R})$, and hence by the Borel Density Theorem [3] that $A_{g}$ is Zariski dense in $G(\mathbb{R})$, so also in $G(\mathbb{C})$.

Consider the fibration sequence

$$
B \widetilde{\mathfrak{T o r}}_{g, 1} \longrightarrow{\widetilde{\operatorname{Diff}_{\partial}}}_{(}\left(W_{g, 1}\right) \longrightarrow B A_{g}
$$

where $B \widetilde{\mathfrak{T o r}}_{g, 1}$ is defined to be the homotopy fibre. By [2, Proposition 4.1] we have

$$
H^{1}\left(B \widetilde{\mathfrak{T o r}}_{g, 1} ; \mathbb{Q}\right) \cong \begin{cases}H_{g} & n \equiv 3 \bmod 4 \\ 0 & \text { else }\end{cases}
$$

so if $n \not \equiv 3 \bmod 4$ then $H^{1}\left(A_{g} ; U\right) \rightarrow H^{1}\left(\widetilde{B \widetilde{\operatorname{Diff}}_{\partial}}\left(W_{g, 1}\right) ; U\right)$ is an isomorphism, and if $n \equiv 3 \bmod 4$ then we have an exact sequence

$$
0 \longrightarrow H^{1}\left(A_{g} ; U\right) \longrightarrow H^{1}\left(\widetilde{\operatorname{Diff}}_{\partial}\left(W_{g, 1}\right) ; U\right) \longrightarrow\left(H_{g} \otimes U\right)^{A_{g}}
$$

In the case $n \equiv 3 \bmod 4, n$ is odd and Zariski density of $A_{g} \leq S p_{2 g}(\mathbb{C})$ implies

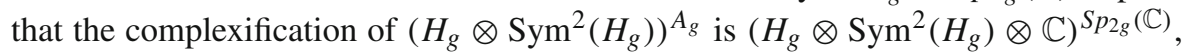
which is contained in $\left(H_{g}^{\otimes 3} \otimes \mathbb{C}\right)^{S p_{2 g}(\mathbb{C})}$ and so vanishes by standard invariant theory (for which we refer to [11, §F.2]).

It remains to show that $H^{1}\left(A_{g} ; U\right)=0$. The representation $U$ is arithmetic, so a theorem of Borel [4, Theorem 1] can be used to identify this with $H^{1}\left(A_{g} ; \mathbb{Q}\right) \otimes U^{A_{g}}$ as long as $g \gg 0$; see [9, Proposition 3.9] for a statement of this result adapted to our situation. Hence it is enough to show the vanishing of $U^{A_{g}}$.

If $n$ is odd then $U^{A_{g}}$ is $\operatorname{Sym}^{2}\left(H_{g}\right)^{A_{g}}$, whose complexification is the same as $\operatorname{Sym}^{2}\left(H_{g} \otimes \mathbb{C}\right)^{S p_{2 g}(\mathbb{C})}$ by Zariski density, and this vanishes by standard invariant the-

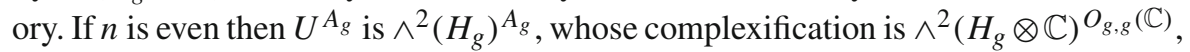
which also vanishes by standard invariant theory (noting that $O_{g, g}(\mathbb{C}) \cong O_{2 g}(\mathbb{C})$ ). 


\section{Characteristic classes of block bundles}

We should like to give a proof of Proposition B, as it does not require the entire corpus $[1,2,12,13]$ and beyond to see that the kernel $(0.3)$ is non-trivial. We shall show that this kernel is non-trivial by producing an explicit element in it, which will be described in terms of generalised Mumford-Morita-Miller classes. If $(\pi: E \rightarrow|K|, \mathcal{A})$ is a smooth oriented block bundle with fibre a closed $d$-manifold $M$ (we refer to [8, Section 2] for this notation), in [8, Section 3] Ebert and the author have associated to it

(i) a Leray-Serre spectral sequence $H^{p}\left(|K|, \mathcal{H}^{q}(M)\right) \Rightarrow H^{p+q}(E)$, and hence a fibre-integration map $\pi_{!}(-): H^{k+d}(E) \rightarrow H^{k}(|K|)$,

(ii) a transfer map $\operatorname{trf}_{\pi}^{*}: H^{*}(E) \rightarrow H^{*}(|K|)$ of Becker-Gottlieb type,

(iii) a stable vertical tangent bundle $T_{\pi}^{s} E \rightarrow E$,

such that if ( $\pi: E \rightarrow|K|, \mathcal{A}$ ) arises from a smooth fibre bundle then these data reduce to those coming from the bundle structure. In the case $d=2 n$, we then employed the following ruse: If $\pi$ came from a smooth fibre bundle with $2 n$-dimensional fibres, so there was an unstable vertical tangent bundle $T_{\pi} E$, then we would have $e\left(T_{\pi} E\right)^{2}=$ $p_{n}\left(T_{\pi} E\right)$, and $\pi_{!}\left(e\left(T_{\pi} E\right) \cdot-\right)=\operatorname{trf}_{\pi}^{*}(-): H^{*}(E) \rightarrow H^{*}(|K|)$. Therefore, for a monomial $p_{I}$ in Pontrjagin classes, if we define

$$
\tilde{\kappa}_{p_{I}}(\pi):=\pi_{!}\left(p_{I}\left(T_{\pi}^{s} E\right)\right) \quad \tilde{\kappa}_{e p_{I}}:=\operatorname{trf}_{\pi}^{*}\left(p_{I}\left(T_{\pi}^{s} E\right)\right)
$$

then these classes restrict to the usual $\kappa_{p_{I}}$ and $\kappa_{e p_{I}}$ on fibre bundles, and these give all generalised Mumford-Morita-Miller classes on fibre bundles.

By way of apology for this ruse, we add to the list above

(iv) an Euler class $e\left(T_{\pi} E\right) \in H^{d}(E ; \mathbb{Z})$.

(Of course $e\left(T_{\pi} E\right)$ is merely notation: there is no $d$-dimensional bundle $T_{\pi} E$ of which it is the Euler class.) Using this Euler class, we may then define

$$
\tilde{\kappa}_{e^{i} p_{I}}(\pi):=\pi !\left(e\left(T_{\pi} E\right)^{i} \cdot p_{I}\left(T_{\pi}^{s} E\right)\right) \in H^{*}(|K| ; \mathbb{Z}) .
$$

The symbol $\tilde{\kappa}_{e p_{I}}$ has the same meaning as before, by Lemma 2.2 (iv) below.

The existence of this Euler class is a consequence of the Fibre Inclusion Theorem of [7] (or rather its proof, which constructs a canonical such class), and the fact that the homotopy fibre of $\pi$ is homotopy equivalent to a Poincaré duality space of dimension $d$, namely $M$ [8, Proposition 2.8]. As the construction is quite pretty, let us describe it.

Construction 2.1 Embed $|K|$ into $\mathbb{R}^{k}$ for some $k \gg 0$, and let $B^{\prime}$ be a closed regular neighbourhood, so that there is a retraction $r: B^{\prime} \rightarrow|K|$. Let $B=D\left(B^{\prime}\right)$ be the double of $B^{\prime}$, a closed smooth manifold. This has a retraction $s: D\left(B^{\prime}\right) \rightarrow B^{\prime}$, and let $p: X \rightarrow B$ be the Hurewicz fibration obtained by turning $\pi$ into a fibration $\pi^{f}: E^{f} \rightarrow|K|$ and pulling it back along $r s$. As $B$ and the fibre of $p$ are Poincaré duality spaces, of dimensions $k$ and $d$ respectively, $X$ is too [14], of dimension $(d+k)$. But $X \times_{B} X=p^{*}(X) \rightarrow X$ is also a fibration over a Poincaré duality space with 
Poincaré duality fibre, so is again a Poincaré duality space, of dimension $(2 d+k)$. Writing $\Delta: X \rightarrow X \times{ }_{B} X$ for the fibrewise diagonal map, which admits an umkehr map $\Delta$ ! as source and target are both Poincaré, we define

$$
e\left(T_{p} X\right):=\Delta^{*} \Delta_{!}(1) \in H^{d}(X ; \mathbb{Z}) .
$$

We then define $e\left(T_{\pi} E\right)$ by restriction along $\left.E \subset E^{f} \subset X\right|_{B^{\prime}} \subset X$.

It is easy to see that the class so obtained is independent of all choices, and it is shown in [7, §4] that it restricts to the Euler class on the fibre $M$. The definition given in $[7, \S 4]$ seems to differ by a sign, but it does not, by Lemma 2.2 (i) below.

Lemma 2.2 The Euler class defined enjoys the following properties:

(i) If $d$ is odd then $2 e\left(T_{\pi} E\right)=0 \in H^{*}(E ; \mathbb{Z})$,

(ii) if $(\pi: E \rightarrow|K|, \mathcal{A})$ arises from a smooth fibre bundle with vertical tangent bundle $T_{\pi} E$, then $e\left(T_{\pi} E\right)$ agrees with the Euler class of the vertical tangent bundle,

(iii) if there is a map $r: E \rightarrow M$ such that $\pi \times r: E \rightarrow|K| \times M$ is a homotopy equivalence, then $e\left(T_{\pi} E\right)=r^{*}(e(T M))$,

(iv) the equation $\pi_{!}\left(e\left(T_{\pi} E\right) \cdot-\right)=\operatorname{trf}_{\pi}^{*}(-): H^{*}(E ; \mathbb{Z}) \rightarrow H^{*}(|K| ; \mathbb{Z})$ is satisfied.

Proof For (i), consider the involution $\tau$ of $X \times_{B} X$ which interchanges the two factors. When $d$ is odd, this has degree -1 , and so $\tau^{*} \Delta_{!}=-\Delta !$. On the other hand $\Delta^{*} \tau^{*}=\Delta^{*}$, so $e\left(T_{\pi} E\right)=-e\left(T_{\pi} E\right)$.

For (ii), note that if $(\pi: E \rightarrow|K|, \mathcal{A}$ ) arises from a smooth fibre bundle then in Construction 2.1 we do not need to replace it by a fibration. The resulting $p: X \rightarrow B$ is a smooth fibre bundle with vertical tangent bundle $T_{p} X$, and the map $\Delta: X \rightarrow X \times{ }_{B} X$ is a smooth embedding with normal bundle $T_{p} X$. Hence $\Delta^{*} \Delta_{!}(1)$ is the Euler class of $T_{p} X$, which restricts to the Euler class of $T_{\pi} E$.

For (iii), if such an $r$ exists then the fibration $p: X \rightarrow B$ admits a similar fibre homotopy trivialisation, $p \times \rho: X \stackrel{\sim}{\rightarrow} B \times M$. Then $X \times_{B} X \simeq B \times M \times M$ and the map $\Delta$ is given by the identity map on $B$ and the diagonal map on $M$. Hence $\Delta^{*} \Delta_{!}(1)=1 \otimes e(T M)$.

For (iv), we must involve ourselves in the details of the construction of the transfer in [7], with which we assume the reader is familiar. We begin by constructing a commutative diagram

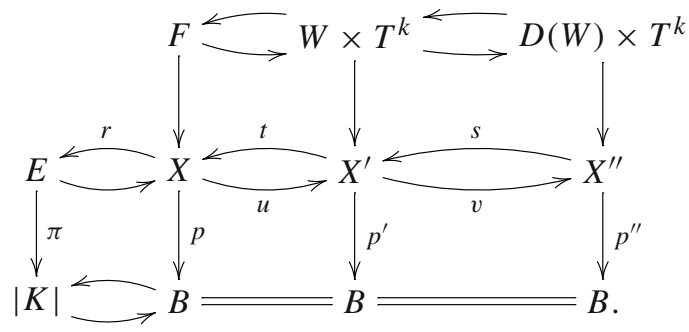

In this diagram, $B$ is a Poincare duality space and $p$ is a Hurewicz fibration with fibre $F \simeq M^{d}$ (obtained as in Construction 2.1). W is a smooth oriented manifold 
of dimension $(d+\ell)$ with boundary, which is homotopy equivalent to $M$, and $p^{\prime}$ is a smooth fibre bundle (obtained from the Closed Fibre Smoothing Theorem of [7]). The map $p^{\prime \prime}$ is obtained as the fibrewise double of $p^{\prime}$, and is a smooth oriented fibre bundle with closed fibres. Finally, the horizontal arrows express each left-hand space as a (fibrewise) retract of the right-hand space.

For a fibration $p: S \rightarrow T$ with fibre homotopy equivalent to a finite $\mathrm{CW}$ complex, and a fibrewise map $f: S \rightarrow S$, let us write $\operatorname{trf}_{p, f}^{*}: H^{*}(S) \rightarrow H^{*}(T)$ for the associated transfer map. This is the map denoted $\tau^{f}$ in [7]. When $f=\operatorname{Id}_{S}$, we shorten this to $\operatorname{trf}_{p}^{*}$.

By the definition of the transfer in $[7, \S 6]$, we have $\operatorname{trf}_{p}^{*}=\operatorname{trf}_{p^{\prime \prime}, v u t s}^{*} s^{*} t^{*}$. By the construction of the transfer for smooth fibre bundles in $[7, \S 5]$, if we write

$$
\begin{aligned}
\delta & =\left(\operatorname{Id}_{X^{\prime \prime}}, \text { vuts }\right): X^{\prime \prime} \longrightarrow X^{\prime \prime} \times_{B} X^{\prime \prime} \\
d & =\left(\operatorname{Id}_{X^{\prime \prime}}, \operatorname{Id}_{X^{\prime \prime}}\right): X^{\prime \prime} \longrightarrow X^{\prime \prime} \times_{B} X^{\prime \prime}
\end{aligned}
$$

then we have trf ${ }_{p^{\prime \prime}, \text { vuts }}^{*}(-)=p_{!}^{\prime \prime}\left(\delta^{*}\left(d_{!}(1)\right) \cdot-\right)$. Thus the map $\operatorname{trf}_{p}^{*}(-)$ is $p_{!}^{\prime \prime}\left(\delta^{*}\left(d_{!}(1)\right)\right.$. $\left.s^{*} t^{*}(-)\right)=(p t s)_{!}\left(\delta^{*}\left(d_{!}(1)\right) \cdot s^{*} t^{*}(-)\right)$, which we may write as $p_{!}\left((t s)_{!}\left(\delta^{*}\left(d_{!}(1)\right)\right.\right.$. $-)$, so we will be done if $(t s) !\left(\delta^{*}\left(d_{!}(1)\right)\right)$ is equal to the class $e\left(T_{p} X\right)$ defined by Construction 2.1. Consider the homotopy cartesian squares
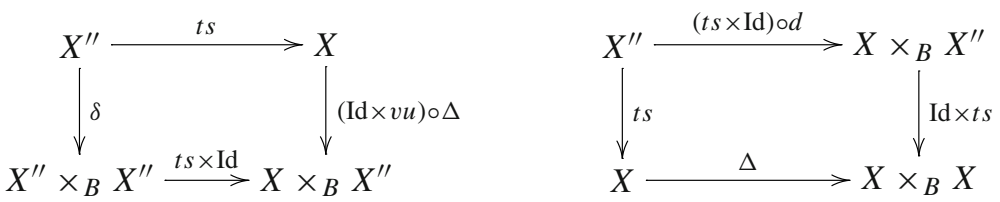

of Poincaré duality spaces, to which Lemma 2.3 below applies and shows that

$$
(t s) ! \delta^{*}=\Delta^{*}(\operatorname{Id} \times v u)^{*}(t s \times \operatorname{Id}) ! \quad(t s \times \operatorname{Id}) ! d_{!}(t s)^{*}=(\operatorname{Id} \times t s)^{*} \Delta_{!} .
$$

(The signs can be determined by restricting each square to a single fibre over $B$.) Thus, writing $1=(t s)^{*}(1)$, we have

$$
\begin{aligned}
(t s) ! \delta^{*} d_{!}(t s)^{*}(1) & =\Delta^{*}(\mathrm{Id} \times v u)^{*}(t s \times \mathrm{Id}) ! d_{!}(t s)^{*}(1) \\
& =\Delta^{*}(\mathrm{Id} \times v u)^{*}(\operatorname{Id} \times t s)^{*} \Delta_{!}(1)=\Delta^{*} \Delta_{!}(1)
\end{aligned}
$$

which is $e\left(T_{p} X\right)$, as required.

Lemma 2.3 Consider a homotopy cartesian square

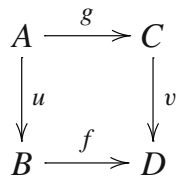

of oriented Poincaré duality spaces. Then $g ! u^{*}= \pm v^{*} f !$. 
The sign ambiguity is unavoidable under the given hypotheses: changing the orientation of $B$, say, does not change $g_{!} u^{*}$, but changes $v^{*} f_{!}$by a sign.

Proof Let us write $a$ for the formal dimension of $A$, and so on. We assume some familiarity with the notion of Poincaré embeddings, for which we refer to [17] for details. It is enough to prove the identity for the larger square

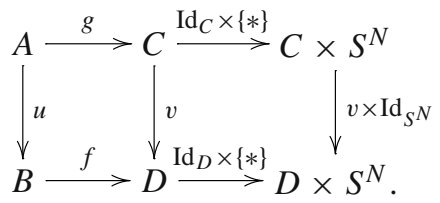

By this device, we may suppose [17, Lemma 3.1] that $f$ admits the structure of a Poincaré embedding, with complement $K$ and normal spherical fibration $\xi$ of dimension $(d-b-1)$. Let $u^{*} \xi \rightarrow A$ denote the pulled back spherical fibration, and $v^{*} K \rightarrow C$ denote the homotopy pullback of the map $K \rightarrow D$ along $v$. There is then a homotopy commutative cube

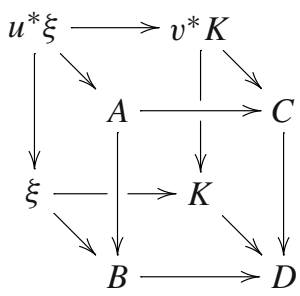

in which the bottom face is homotopy cocartesian, and the vertical faces are all homotopy cartesian. It follows by Mather's Second Cube Theorem [18, Theorem 25] that the top face is also homotopy cocartesian. We therefore have a map

$$
C \simeq A \cup_{u^{*} \xi} v^{*} K \longrightarrow A / u^{*} \xi=\operatorname{Th}\left(u^{*} \xi\right)
$$

by collapsing $v^{*} K$, and similarly for $K$. This gives a homotopy commutative diagram

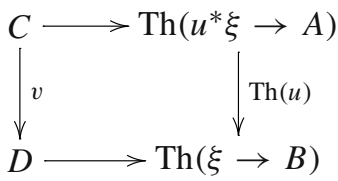

which in cohomology yields the required equation. From this point of view, the sign ambiguity arises from the two possible choices of Thom class for $u^{*} \xi$ : the one compatible with the fundamental classes $[C]$ and $[A]$, or the pullback of the one compatible with $[D]$ and $[B]$. 


\section{Proof of Proposition B}

We can extend the definition of the classes $\tilde{\kappa}_{e^{i}} p_{I}$ to block bundles having fibres $W_{g, 1}$ by filling in a disc in each fibre, giving a new block bundle with fibre $W_{g}:=W_{g, 1} \cup_{\partial}$ $D^{2 n}=\#^{g} S^{n} \times S^{n}$. There are therefore defined universal characteristic classes $\tilde{\kappa}_{e^{i} p_{I}} \in$ $H^{*}\left(\widetilde{B \operatorname{Diff}_{\partial}}\left(W_{g, 1}\right) ; \mathbb{Q}\right)$, by the proof of [8, Theorem 3.4].

In particular, we have a class $\tilde{\kappa}_{e^{2}}-\tilde{\kappa}_{p_{n}} \in H^{2 n}\left(B \widetilde{\operatorname{Diff}}{ }_{\partial}\left(W_{g, 1}\right) ; \mathbb{Q}\right)$ which vanishes in $H^{2 n}\left(B \operatorname{Diff}_{2}\left(W_{g, 1}\right) ; \mathbb{Q}\right)$, because $e^{2}=p_{n}$ on the total space of a smooth fibre bundle. Proposition $\mathrm{B}$ is an immediate consequence of the following.

Proposition 3.1 For each $g \geq 1$ and each $n \geq 3$ there is a block bundle ( $\pi: E \rightarrow$ $|K|, \mathcal{A})$ with fibre $W_{g, 1}$, such that

(i) $\tilde{\kappa}_{e^{2}}(\pi)=0 \in H^{2 n}(|K| ; \mathbb{Q})$,

(ii) $\tilde{\kappa}_{p_{n}}(\pi) \neq 0 \in H^{2 n}(|K| ; \mathbb{Q})$.

Therefore $\tilde{\kappa}_{e^{2}}-\tilde{\kappa}_{p_{n}} \neq 0 \in H^{2 n}\left(\widetilde{B \operatorname{Diff}_{\partial}}\left(W_{g, 1}\right) ; \mathbb{Q}\right)$.

Proof From Lemma 2.2 (iii) it follows that the $\tilde{\kappa}_{e^{i}}$ vanish for all $i>0$ on all fibre homotopically trivial block bundles. We will therefore construct $\pi$ to be fibre homotopically trivial, guaranteeing that $\tilde{\kappa}_{e^{2}}(\pi)=0$.

We will use the (space-level) surgery fibration of Quinn [19], which following the discussion in [1, Section 3.2], in particular equation (43), may be put in the form

$$
\left(\frac{\operatorname{hAut}_{\partial}\left(W_{g, 1}\right)}{\widehat{\operatorname{Diff}}_{\partial}\left(W_{g, 1}\right)}\right)_{(1)} \longrightarrow \operatorname{map}_{*}\left(W_{g, 1} / \partial W_{g, 1}, G / O\right)_{(1)} \stackrel{\sigma}{\longrightarrow} \mathbb{L}_{2 n}(\mathbb{Z})_{(1)}
$$

Thus to construct a fibre homotopically trivial block bundle over $B$ (with some triangulation) it is enough to give a map $f: B \rightarrow \operatorname{map}_{*}\left(W_{g, 1} / \partial W_{g, 1}, G / O\right)_{(1)}$ and a nullhomotopy of $\sigma \circ f$.

For simplicity of exposition we restrict to the case $n=2 k$. We let $B=S^{n} \times$ $S^{n}$, write $a, b \in H^{n}(B ; \mathbb{Q})$ for a hyperbolic basis, and write $e_{1}, f_{1}, \ldots, e_{g}, f_{g} \in$ $H^{n}\left(W_{g, 1}, \partial W_{g, 1} ; \mathbb{Q}\right)$ for a hyperbolic basis. Write the $n$th Hirzebruch $L$-polynomial as $\mathcal{L}_{n}=A p_{n}+B p_{n / 2}^{2}$ modulo other Pontrjagin classes, for some constants $A$ and $B$. It is well-known that $A \neq 0$, and less well-known but true [21, Lemma A.1] that $B \neq 0$.

As the composition

$$
p: G / O \stackrel{i}{\longrightarrow} B O \stackrel{\prod p_{i}}{\longrightarrow} \prod_{i=1}^{\infty} K(\mathbb{Z}, 4 i)
$$

has homotopy fibre with finite homotopy groups, we claim that may find a map $f$ whose adjoint $\hat{f}:\left(B \times W_{g, 1}, B \times \partial W_{g, 1}\right) \rightarrow(G / O, *)$ composed with $i$ gives a class

$$
\xi \in K O^{0}\left(B \times W_{g, 1}, B \times \partial W_{g, 1}\right)
$$


which has $p_{n / 2}(\xi)=C \cdot\left(a \otimes e_{1}+b \otimes f_{1}\right), p_{n}(\xi)=-\frac{2 B C^{2}}{A} \cdot a \cdot b \otimes e_{1} \cdot f_{1}$, and all other rational Pontrjagin classes zero, for some constant $C \neq 0$. To establish this claim, let the map

$$
\varphi:\left(B \times W_{g, 1}, B \times \partial W_{g, 1}\right) \longrightarrow\left(\prod_{i=1}^{\infty} K(\mathbb{Z}, 4 i), *\right)
$$

classify the pair of relative cohomology classes $L \cdot\left(a \otimes e_{1}+b \otimes f_{1}\right)$ and $-\frac{2 B L^{2}}{A} \cdot a$. $b \otimes e_{1} \cdot f_{1}$, for some integer $L \neq 0$ large enough that these classes are integral. For each $N>0$ consider the map $\phi_{N}: \prod_{i} K(\mathbb{Z}, 4 i) \rightarrow \prod_{i} K(\mathbb{Z}, 4 i)$ which multiplies by $N^{i}$ on $K(\mathbb{Z}, 4 i)$. The diagram

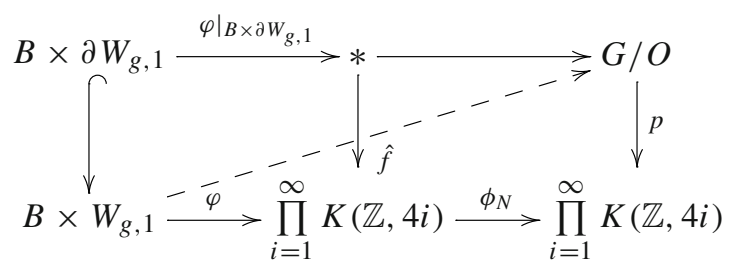

then admits a dotted lift $\hat{f}$ for $N$ large enough, as the universal obstructions to finding such a lift lie in the cohomology of $\prod_{i} K(\mathbb{Z}, 4 i)$ with finite coefficients, and are therefore annihilated (on each skeleton) by some $\phi_{N}$. The resulting map $\hat{f}$ gives $p_{n / 2}(\xi)=L \cdot N^{n / 2} \cdot\left(a \otimes e_{1}+b \otimes f_{1}\right), p_{n}(\xi)=-\frac{2 B L^{2}}{A} \cdot N^{n} \cdot a \cdot b \otimes e_{1} \cdot f_{1}$, and all other Pontrjagin classes zero, as required (with $C=L \cdot N^{n / 2}$ ).

We must show that the composition

$$
B=S^{n} \times S^{n} \stackrel{f}{\longrightarrow} \operatorname{map}_{*}\left(W_{g, 1} / \partial W_{g, 1}, G / O\right)_{(1)} \stackrel{\sigma}{\longrightarrow} \mathbb{L}_{2 n}(\mathbb{Z})_{(1)}
$$

is nullhomotopic, but we shall allow ourselves to precompose $f$ with self-maps $k_{N}$ : $S^{n} \times S^{n} \rightarrow S^{n} \times S^{n}$ having degree $N \neq 0$ on both factors (such a precomposition preserves the form of Pontrjagin classes which has been arranged above). With this in mind, it is enough to show that

$$
\sigma \circ f=0 \in\left[B, \mathbb{L}_{2 n}(\mathbb{Z})\right] \otimes \mathbb{Q}
$$

This group may be identified with $H^{4 *}(B ; \mathbb{Q})$. If $n \equiv 0 \bmod 4$ then the component of degree $n=2 k=4 \ell$ is identified with the Künneth factor of 


$$
\frac{1}{8} \mathcal{L}_{3 \ell}(\xi) \in H^{12 \ell}\left(B \times W_{g, 1}, B \times \partial W_{g, 1} ; \mathbb{Q}\right)
$$

in $H^{4 \ell}(B ; \mathbb{Q}) \cong H^{4 \ell}(B ; \mathbb{Q}) \otimes H^{8 \ell}\left(W_{g, 1}, \partial W_{g, 1} ; \mathbb{Q}\right)$. But $\mathcal{L}_{3 \ell}(\xi)=0$ by observation, as only $p_{4 \ell}(\xi)$ and $p_{2 \ell}(\xi)$ are non-zero. Whatever the class of $n$ modulo 4 , the component of degree $2 n=4 k$ is identified with the Künneth factor of

$$
\frac{1}{8} \mathcal{L}_{2 k}(\xi) \in H^{8 k}\left(B \times W_{g, 1}, B \times \partial W_{g, 1} ; \mathbb{Q}\right)
$$

in $H^{4 k}(B ; \mathbb{Q})$. But by construction

$$
\mathcal{L}_{2 k}(\xi)=A \cdot\left(-\frac{2 B C^{2}}{A} \cdot a \cdot b \otimes e_{1} \cdot f_{1}\right)+B \cdot\left(C \cdot\left(a \otimes e_{1}+b \otimes f_{1}\right)\right)^{2}=0 .
$$

We therefore obtain a map $f$, with $\sigma \circ f$ nullhomotopic and $i \circ \hat{f}$ classifying a vector bundle $\xi^{\prime}$ having $p_{n / 2}\left(\xi^{\prime}\right)=D \cdot\left(a \otimes e_{1}+b \otimes f_{1}\right), p_{n}\left(\xi^{\prime}\right)=-\frac{2 B D^{2}}{A} \cdot a \cdot b \otimes e_{1} \cdot f_{1}$, and all other Pontrjagin classes zero, for some constant $D \neq 0$. (The constant will have changed when we precomposed the original choice of $f$ with the maps $k_{N}$.) The associated block bundle $\pi: E \rightarrow|K| \approx B$ has $T_{v}^{s} E \simeq \simeq_{s} T E-\pi^{*} T B=\epsilon^{2 n}+\xi^{\prime}$ (see [8, Lemma 3.3]) and so

$$
\tilde{\kappa}_{p_{n}}(\pi)=\pi_{!}\left(p_{n}\left(T_{v}^{s} E\right)\right)=\pi_{!}\left(p_{n}\left(\xi^{\prime}\right)\right)=-\frac{2 B D^{2}}{A} \cdot a \cdot b \neq 0
$$

as required.

It is not difficult to adapt the above argument to work for $n=2 k+1$. The essential point is that if we write $\mathcal{L}_{n}=A p_{n}+B p_{\frac{n-1}{2}} p_{\frac{n+1}{2}}$ modulo all other Pontrjagin classes, then $A \neq 0$ and again by [21, Lemma A.1] $B \neq 0$. We then take $B=S^{2 k-1} \times S^{2 k+3}$ and proceed as above.

\section{Rational connectivity of $\operatorname{Top}(2 n) / O(2 n)$}

Our goal in this section is to show how similar techniques to those we have been using imply the following.

Theorem $4.1 \pi_{*}\left(B \operatorname{Diff}_{\partial}\left(D^{2 n}\right)\right) \otimes \mathbb{Q}=0$ for $1 \leq * \leq 2 n-5$.

This extends the analogous calculation of Farrell-Hsiang [10], which established the same result in degrees $1 \leq * \leq \phi(2 n)$. By smoothing theory we have a homotopy equivalence $B \operatorname{Diff}_{\partial}\left(D^{2 n}\right) \simeq \Omega_{0}^{2 n}(\operatorname{Top}(2 n) / \mathrm{O}(2 n))$ as long as $2 n>4$, from which we deduce that

Corollary 4.2 $\mathrm{Top}(2 n) / \mathrm{O}(2 n)$ is rationally $(4 n-5)$-connected as long as $n>2$.

On the other hand, it has been shown by Weiss [21] that

$$
H^{4 n}(B \operatorname{Top}(2 n) ; \mathbb{Q}) \longrightarrow H^{4 n}(B \mathrm{O}(2 n) ; \mathbb{Q})
$$


has nontrivial kernel for $n \gg 0$ (namely, the class $e^{2}-p_{n}$ ), so $\operatorname{Top}(2 n) / \mathrm{O}(2 n)$ is not rationally $(4 n-1)$-connected.

Proof of Theorem 4.1 Let $W_{g}=\#^{g} S^{n} \times S^{n} \backslash \operatorname{int}\left(D^{2 n}\right)$, with $2 n \geq 6$, and choose a collar $[0,1) \times \partial W_{g, 1} \subset W_{g, 1}$ and a disc $D^{2 n} \subset(0,1) \times \partial W_{g, 1}$. The map

$$
\frac{\widetilde{\operatorname{Diff}_{\partial}}\left(D^{2 n}\right)}{\operatorname{Diff}_{\partial}\left(D^{2 n}\right)} \longrightarrow \frac{\widetilde{\operatorname{Diff}_{\partial}}\left(W_{g, 1}\right)}{\operatorname{Diff}_{\partial}\left(W_{g, 1}\right)}
$$

is $(2 n-4)$-connected, by Morlet's lemma of disjunction [6, Corollary 3.2]. Consider the fibration

$$
\frac{\widetilde{\operatorname{Diff}_{\partial}}\left(W_{g, 1}\right)}{\operatorname{Diff}_{\partial}\left(W_{g, 1}\right)} \longrightarrow B \operatorname{Diff}_{\partial}\left(W_{g, 1}\right) \stackrel{i}{\longrightarrow} \widetilde{\operatorname{Diff}}_{\partial}\left(W_{g, 1}\right)
$$

Up to isotopy any diffeomorphism $\varphi$ representing an element of $\pi_{1}\left(\widetilde{B \operatorname{Diff}_{\partial}}\left(W_{g, 1}\right)\right)$ may be supposed to be equal to the identity on the collar: the map (4.1) is then preserved by that induced by $\varphi$, and it then follows that $\varphi$ acts trivially on $H^{*}\left(\widetilde{\operatorname{Diff}_{\partial}\left(W_{g, 1}\right)} ; \mathbb{\operatorname { D i f f } _ { \partial } ( W _ { g , 1 } )} ;\right)$ in the range of degrees $* \leq 2 n-4$ where the map (4.1) is a cohomology injection. Hence the Serre spectral sequence

$$
H^{p}\left(\widetilde{\operatorname{Diff}_{\partial}}\left(W_{g, 1}\right) ; H^{q}\left(\widetilde{\operatorname{Diff}_{\partial}\left(W_{g, 1}\right)} ; \mathbb{Q}\right)\right) \Longrightarrow H^{p+q}\left(B \operatorname{Diff}_{\partial}\left(W_{g, 1}\right) ; \mathbb{Q}\right)
$$

associated to this fibration has a product structure in this range of degrees. But Berglund-Madsen have shown that the map

$$
i^{*}: H^{*}\left(\widetilde{B \operatorname{Diff}_{\partial}}\left(W_{g, 1}\right) ; \mathbb{Q}\right) \longrightarrow H^{*}\left(B \operatorname{Diff}_{\partial}\left(W_{g, 1}\right) ; \mathbb{Q}\right)
$$

is an isomorphism in degrees $* \leq 2 n-1$ as long as $g \gg 0$. This result will appear soon in a revision of [2]. It follows that $H^{q}\left(\frac{\widetilde{\operatorname{Diff}_{\partial}}\left(W_{g, 1}\right)}{\overline{\operatorname{Diff}}_{\partial}\left(W_{g, 1}\right)} ; \mathbb{Q}\right)=0$ for $1 \leq q \leq 2 n-4$, and hence by the map (4.1) that $H^{q}\left(\frac{\widetilde{\operatorname{Diff}_{\partial}}\left(D^{2 n}\right)}{\operatorname{Diff}_{\partial}\left(D^{2 n}\right)} ; \mathbb{Q}\right)=0$ for $1 \leq q \leq 2 n-5$.

On the other hand, the surgery fibration sequence shows that $\frac{\mathrm{hAut}_{\partial}\left(D^{2 n}\right)}{\operatorname{Diff}_{\partial}\left(D^{2 n}\right)}$ is rationally acyclic, and $\operatorname{hAut}_{\partial}\left(D^{2 n}\right) \simeq *$ by the Alexander trick, so $\widetilde{B \widetilde{D i f f}_{\partial}}\left(D^{2 n}\right)$ is rationally acyclic. Boundary connect-sum makes this into an $H$-space, so it has trivial rational homotopy groups. Thus $\widetilde{\operatorname{Diff}_{\partial}}\left(D^{2 n}\right)$ has finitely-many components, and each one is rationally acyclic. The group $\operatorname{Diff}_{\partial}\left(D^{2 n}\right)$ has the same components, and so the quotient

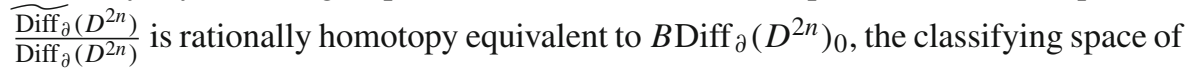
the component of the identity in $B \operatorname{Diff}_{\partial}\left(D^{2 n}\right)$. It follows from the above that its rational cohomology, and hence rational homotopy, vanishes in degrees $1 \leq * \leq 2 n-5$. 
Acknowledgements I am grateful to Alexander Berglund and to Ib Madsen for (repeatedly) explaining to me their forthcoming results. The author was supported by EPSRC Grant EP/M027783/1, and is grateful to Stanford University for its hospitality while this paper was written.

Open Access This article is distributed under the terms of the Creative Commons Attribution 4.0 International License (http://creativecommons.org/licenses/by/4.0/), which permits unrestricted use, distribution, and reproduction in any medium, provided you give appropriate credit to the original author(s) and the source, provide a link to the Creative Commons license, and indicate if changes were made.

\section{References}

1. Berglund, A., Madsen, I.: Homological stability of diffeomorphism groups. Pure Appl. Math. Q. 9(1), 1-48 (2013)

2. Berglund, A., Madsen, I.: Rational homotopy theory of automorphisms of highly connected manifolds (2014). arXiv:1401.4096

3. Borel, A.: Density properties for certain subgroups of semi-simple groups without compact components. Ann. Math. 2(72), 179-188 (1960)

4. Borel, A.: Stable and $L^{2}$-cohomology of arithmetic groups. Bull. Am. Math. Soc. (N.S.), 3(3), 10251027 (1980)

5. Borel, A., Harish-Chandra: Arithmetic subgroups of algebraic groups. Ann. Math. 2(75), 485-535 (1962)

6. Burghelea, D., Lashof, R., Rothenberg, M.: Groups of automorphisms of manifolds. Lecture Notes in Mathematics, vol. 473. Springer, Berlin (1975)

7. Casson, A., Henry Gottlieb, D.: Fibrations with compact fibres. Am. J. Math. 99(1), 159-189 (1977)

8. Ebert, J., Randal-Williams, O.: Generalised Miller-Morita-Mumford classes for block bundles and topological bundles. Algebr. Geom. Topol. 14(2), 1181-1204 (2014)

9. Ebert, J., Randal-Williams, O.: Torelli spaces of high-dimensional manifolds. J. Topol. 8(1), 38-64 (2015)

10. Farrell, F.T., Hsiang, W.C.: On the rational homotopy groups of the diffeomorphism groups of discs, spheres and aspherical manifolds. In: Algebraic and geometric topology (Proc. Sympos. Pure Math., Stanford Univ., Stanford, Calif., 1976), Part 1, Proc. Sympos. Pure Math., vol. XXXII, pp. 325-337. Amer. Math. Soc., Providence (1978)

11. Fulton, W., Harris, J.: Representation theory, Graduate Texts in Mathematics, vol. 129. Springer, New York (1991). A first course, Readings in Mathematics

12. Galatius, S., Randal-Williams, O.: Homological stability for moduli spaces of high dimensional manifolds. I (2014). arXiv:1403.2334

13. Galatius, S., Randal-Williams, O.: Stable moduli spaces of high-dimensional manifolds. Acta Math. 212(2), 257-377 (2014)

14. Henry Gottlieb, D.: Poincaré duality and fibrations. Proc. Am. Math. Soc. 76(1), 148-150 (1979)

15. Hsiang, W.C., Staffeldt, R.E.: A model for computing rational algebraic $K$-theory of simply connected spaces. Invent. Math. 68(2), 227-239 (1982)

16. Igusa, K.: The stability theorem for smooth pseudoisotopies. $K$-Theory $\mathbf{2}(1-2)$, vi+355 (1988)

17. Klein, J.R.: Poincaré duality embeddings and fiberwise homotopy theory. Topology 38(3), 597-620 (1999)

18. Mather, M.: Pull-backs in homotopy theory. Can. J. Math. 28(2), 225-263 (1976)

19. Quinn, F. :A geometric formulation of surgery. In: Topology of Manifolds (Proc. Inst., Univ. of Georgia, Athens, GA, 1969), pp. 500-511, Markham (1970)

20. Waldhausen, F., Jahren, B., Rognes, J.: Spaces of PL manifolds and categories of simple maps. Annals of Mathematics Studies, vol. 186. Princeton University Press, Princeton (2013)

21. Weiss, M.: Dalian notes on Pontryagin classes (2015). arXiv:1507.00153

22. Weiss, M., Williams, B.: Automorphisms of manifolds and algebraic $K$-theory. I. $K$-Theory 1(6), 575-626 (1988) 